\title{
Acceso de mujeres en embarazo, parto y post parto a servicios de salud según clase social
}

\section{Access of women in pregnancy, childbirth and postpartum to health services according to social class}

\author{
Libia-A. Bedoya-Ruiz ${ }^{1}$; Andrés-A. Agudelo-Suárez ${ }^{1,2}$; Diego-A. Restrepo-Ochoa ${ }^{1}$
}

Forma de citar: Bedoya-Ruiz LA, Agudelo-Suárez AA, Restrepo-Ochoa DA. Acceso de mujeres en embarazo, parto y post parto a servicios de salud según clase social. Salud UIS. 2020; 52(3): $285-294$. doi: http://dx.doi.org/10.18273/revsal.v52n3-2020009 (c) (1)

\section{Resumen}

Objetivo: comprender el acceso que tienen las mujeres en situación de embarazo, parto y post parto a los servicios de salud según la clase social en Bogotá (Colombia). Métodos: estudio cualitativo. Perspectiva hermenéutica crítica. Método etnografía crítica. Muestreo teórico. Análisis por triangulación en Atlas Ti. Participaron 9 mujeres y 8 profesionales de la salud. Se realizaron 38 entrevistas a profundidad durante 13 meses y 62 acompañamientos a las maternas en las actividades de control prenatal, vacunación, trabajo de parto, consulta post parto, exámenes de seguimiento, curso Psicoprofiláctico, hospitalización y sala de espera, tanto en servicios públicos como privados. Resultados: existen desigualdades según la clase social en el acceso que tienen las mujeres a los servicios de salud en los siguientes aspectos: acceso a servicio a especialistas, hacer la fila para esperar la atención, la disponibilidad de citas y agenda para programar la cita, perder la cita habiendo llegado al servicio, la prioridad que le dan las instituciones a las maternas, madrugar para conseguir atención, pedir la cita, las condiciones de la espera y elegir la clínica o el personal. Conclusiones: los anteriores aspectos se intensifican en clases sociales con menos ventajas. Se requiere disminuir las desigualdades sociales para disminuir las inequidades en salud.

Palabras clave: Clase Social; Servicios de salud materna; Embarazo; Parto; Periodo Posparto; Accesibilidad a los servicios de salud; Derecho sanitario.

\begin{abstract}
Objective: to understand the access that women in pregnancy, childbirth and postpartum have to health services according to social class in Bogotá (Colombia). Methods: qualitative study. Critical hermeneutical perspective. Critical ethnography method. Theoretical sampling. Analysis by triangulation in Atlas Ti. 9 women and 8 health professionals participated. 38 in-depth interviews were carried out during 13 months and 62 accompaniments to the maternal in the activities of prenatal control, vaccination, labor, postpartum consultation, follow-up examinations, Psychoprophylactic course, hospitalization and waiting room, both in public services as private. Results: there are inequalities according to social class in the access that women have to health services in the following aspects: access to specialist services, queuing to wait for care, availability of appointments and schedule to schedule the appointment, Missing the appointment having arrived at the service, the priority that institutions give to maternal

1. Universidad CES. Medellín, Colombia.

2. Universidad de Antioquia. Medellín, Colombia.

Correspondencia: Libia A Bedoya-Ruiz. Dirección: Universidad CES - Calle 10A 22-04. Teléfono (+574) 4440555 Extensión 1745. Correo electrónico: bedoyalibia@hotmail.com
\end{abstract}


mothers, getting up early to get care, requesting an appointment, waiting conditions and choosing the clinic or staff. Conclusions: the previous aspects are intensified in social classes with less advantages. Reducing social inequalities is required to decrease health inequities.

Keywords: Social class; Maternal health services; Pregnancy; Parturition; Postpartum period; Health services accessibility; Health law.

\section{Introducción}

La salud materna está contemplada dentro de los objetivos del Plan Decenal de Salud Pública 20122021 para el país. Se promueven acciones, como el abordaje integral de la mujer antes durante y después del parto, la humanización de los servicios de salud donde se incorpore el enfoque de derecho, eliminación de barreras de acceso a los servicios de salud, y el fortalecimiento institucional ${ }^{1}$.

La ciudad de Bogotá ha sido una de las zonas en Colombia que ha mostrado avances en términos de mortalidad materna ${ }^{2,3}$. A pesar de lo anterior, continua siendo éste un problema de salud pública ${ }^{1}$ donde se ha identificado, que no sólo son importantes las causas de mortalidad materna derivadas del embarazo (causas obstétricas), sino también las causas no obstétricas ${ }^{4}$ como las barreras de acceso geográfico a los servicios de salud, el déficit estructural de las instituciones sanitarias (falta de insumos médicos, deficiencia de personal capacitado, negligencia en la atención) y aspectos sociales y culturales relacionados con la madre (bajo nivel educativo y socioeconómico) $)^{5}$. Otra problemática son las desigualdades sociales, pues la mortalidad materna se concentra en algunas de las localidades del sur más pobres de la capital ${ }^{6}$.

Por ello, se deben promover procesos investigativos que permitan comprender mejor el problema relacionado con las causas no obstétricas ${ }^{4}$ y plantear estrategias en salud pública que ayuden a fortalecer la salud materna desde un punto de vista social, donde las acciones no solo se encaminen a prevenir el problema desde el punto de vista biológico y epidemiológico; sino que también se incorpore el punto de vista político que le permita a las personas de bajos recursos mejorar sus condiciones de vida ${ }^{7}$. Es importante asegurar el derecho fundamental a la salud donde las instituciones sanitarias cuenten con la infraestructura necesaria para resolver los problemas relacionados con el embarazo parto y post parto ${ }^{8}$.

Los antecedentes sobre el tema fueron publicados previamente en una revisión narrativa de la literatura ${ }^{9}$, donde se expusieron los vacíos en investigación que fundamentan el objetivo de la presente investigación. Este objetivo es comprender el acceso que tienen las mujeres en situación de Embarazo, Parto, y Post parto (EPP) a los servicios de salud según la clase social en Bogotá (Colombia).

\section{Enfoque teórico metodológico}

Este proyecto se formula a partir de uno de los objetivos específicos de una investigación más amplia denominada "Configuración de la relación de las mujeres en Embarazo Parto y Postparto (EPP) con los servicios de salud en diferentes niveles de atención según la clase social". El método utilizado para todos los objetivos específicos de la anterior investigación fue el mismo y ya fue publicado. En dicha publicación ${ }^{10} \mathrm{se}$ describen con precisión los materiales y métodos que aplican para los resultados del presente artículo.

Se realizó un estudio cualitativo, con una perspectiva hermenéutica crítica, usando el método de la etnografía crítica. El diseño metodológico fue flexible teniendo en cuenta las necesidades de los participantes y las características del trabajo de campo. Se cumplieron los criterios de calidad de la investigación cualitativa como la confiabilidad, transferibilidad, credibilidad, auditabilidad, y consistencia. El muestreo fue teórico según la teoría de clase social de Breilh. Participaron 9 mujeres que tienen diferentes niveles educativos, estratos socioeconómicos, régimen de salud, seguro de salud, y viven en diferentes localidades de Bogotá. Participaron 8 profesionales de salud de diferentes regímenes de salud, seguros de salud y de diferente sexo (Tablas 1, 2). Se realizaron 38 entrevistas a profundidad en total, contando el personal de salud, durante 13 meses. Se realizaron 62 acompañamientos a las maternas en los servicios de salud durante el proceso de EPP, realizando observación participante, la cual fue registrada en el diario campo. Con este insumo se redactaron itinerarios de acompañamiento en relato etnográfico. Se realizó un análisis por triangulación en Atlas Ti que incluyó la perspectiva de los participantes, la observación participante realizada por la investigadora principal y datos estadísticos del contexto de Bogotá. En el presente artículo solo se presentan 2 categorías de 11 categorías que se 
obtuvieron del análisis en Atlas Ti. En la transcripción de las entrevistas y en el análisis de datos participaron, adicional al grupo de investigación, personal de las ciencias humanas ${ }^{10}$.

Los consentimientos informados se obtuvieron previo dialogo con los participantes sobre el diseño de la investigación. Se leyó el documento antes de que los participantes firmaran. Se especificó que los participantes no recibirían remuneración económica con el hecho de participar en la investigación, y que se protegería la identidad e historia de vida de cada uno de ellos. El consentimiento informado fue entregado a los participantes para que fuera revisado con calma con sus redes sociales y así se dio la oportunidad que se manifestaran inquietudes previas sobre el tema. Varios días después de la entrega del documento las personas que libremente quisieron participar firmaron el consentimiento informado.

Se eligieron perspectivas teóricas críticas en concordancia con la postura epistemológica de la hermenéutica crítica ${ }^{11}$. El marco referencial que se planteó previo al trabajo de campo incluyó los conceptos de clase social y acceso a servicios de salud.

Breilh define clase social como "el conjunto de sujetos organizados alrededor de intereses comunes"12 que "se diferencian entre sí por el lugar que ocupan en un sistema de producción determinado, por las relaciones en que se encuentran frente a los medios de producción, por el papel que desempeñan en la organización del trabajo y, por consiguiente, por el modo y la proporción en que perciben la parte de la riqueza social de que disponen"13.

Respecto a la definición de acceso, Abadía desarrolla la categoría "itinerarios burocráticos", explicada por las luchas que entabla la población colombiana para acceder a los servicios de salud. Lo anterior se debe a fallas que tiene el sistema basado en la rentabilidad, imponiendo barreras administrativas donde se niegan tratamientos y otros servicios. Esto trae consecuencias para la vida, generando sufrimiento y complicaciones médicas ${ }^{14}$.

Los anteriores aspectos teóricos fueron incluidos en las entrevistas a profundidad que se realizaron de forma no estructurada. Sin embargo, el marco teórico se construyó en todo el proceso investigativo y los aspectos que fueron emergentes durante el trabajo de campo se expondrán en la discusión.

\section{Resultados}

Se encontraron 2 categorías enunciadas en las figuras 1 y 2.

Clase social en relación con los servicios de salud (Figura 1).

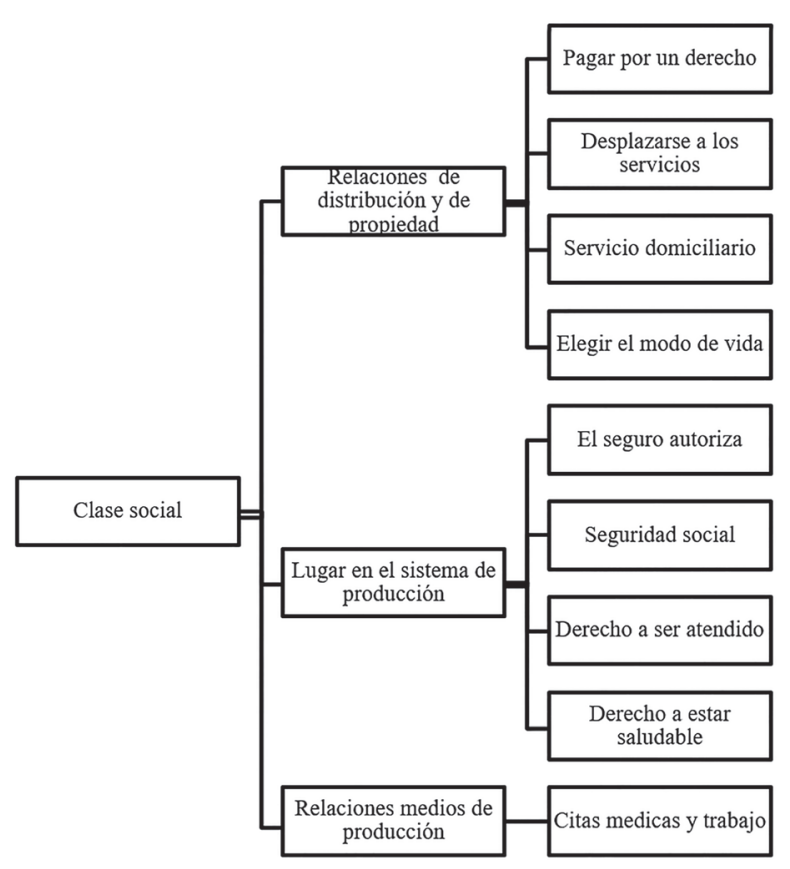

Figura 1. Relación con los servicios según la clase social.

En la conceptualización de Breilh de clase social ${ }^{13}$ hubo subcategorías que se ajustaron más al objetivo planteado. Estas subcategorías son:

Relaciones de distribución (proporción de riqueza) y de propiedad: estas relaciones les permiten a las mujeres elegir los modos de vida, donde se paga por servicios privados de salud que deberían ser derechos, transporte privado para desplazarse en Bogotá a los servicios, y la posibilidad de tener servicio de salud domiciliario.

"por eso fue que adquirí plan complementario, porque realmente queríamos un mejor servicio y mejor atención en el sistema de salud, ya conocíamos por parte de familiares como era el trabajo del parto sin esas condiciones, o sea, por EPS o por Sisbén. Tuve la fortuna, de acompañar a una prima a un parto pero fue en unas condiciones totalmente terribles, o sea ella en una camilla, que yo en ese momento fue que dije si yo quiero un bebé, necesito algo que me ayude a mejorar las condiciones, y pues es triste que uno tenga que pagar por un derecho y por tener esas condiciones dignas que pues es lo mínimo que uno puede pedir, pues 
en el caso de ella que eran cosas como tan elementales hasta de higiene, de salubridad, yo dije no, a mi me dolió muchísimo, entonces afortunadamente con mi embarazo anterior tenía medicina complementaria" (mujer profesional ama de casa plan complementario)

Lugar en el sistema de producción (ocupación, contratación): es el lugar que les permite a las mujeres tener derecho al trabajo y a la seguridad social. Lo anterior se relaciona con la inclusión en un régimen de salud, derecho a la licencia de maternidad y acceso efectivo a servicios de salud lo cual le da a las mujeres el derecho a ser atendidas y estar saludables.

"pero lo básico, saber cómo esta una hemoglobina, un hematocrito, o sea lo que lleva el riesgo con el bebé también me parece terrible, que no se tengan derechos en eso ¿sí?, y que el gobierno se haga lo que no es con ellos, o sea el gobierno no tiene políticas en estos momentos para los que no tienen trabajo, es asi de sencillo, o sea las madres desempleadas no tienen derechos a nada en salud, y ese bebe por lo tanto tampoco" (mujer médica empresaria con prepagada).

Relaciones con los medios de producción (grado de control del proceso de trabajo): se refiere a la formalidad en el trabajo y la relación con los superiores en el ámbito laboral que gestiona la asistencia a los servicios de salud.

"Ninguna actividad del trabajo me estresa. Solo mi jefe. Le pedí permiso para los controles prenatales e hizo como "ahg" esa cara de bueno, yo sé que me toca, pero uish dios, entonces eso como que ya lo indispone un poco" (mujer técnica, asalariada, régimen contributivo).

"ser independiente le permite a uno hacer muchas que realmente si yo creo que si estuviera empleada no lo pudiera hacer, como el cumplimiento, los permisos, la sensación que da uno poder estar un poquito más libre a ciertas horas del día y poder dedicarle más tiempo a otras cosas como el ejercicio, o las cosas que uno quiera hacer para asistir a citas por ejemplo, no son tan agobiantes como si uno tuviera que pedir permiso o hacer digamos el tipo de procesos que tienen que hacer en muchas empresas muchos empleados para poder asistir a una cita médica" ( mujer medica empresaria con prepagada).

Estas subcategorías de clase social se relacionan con las desigualdades en salud según el régimen de afiliación en salud, pues el proceso de trabajo de la población colombiana permite el acceso a bienes $\mathrm{y}$ servicios en las instituciones de salud ${ }^{15}$. Hay mujeres en el régimen subsidiado con trabajo informal, en el régimen contributivo básico mujeres asalariadas, y en el régimen contributivo básico con prepagada una mujer empresaria. Aunque en los dos últimos regímenes está presenta el trabajo doméstico no remunerado, la capacidad de consumo es mayor, al igual que la asistencia a los servicios de salud. Este uso de servicios según la capacidad económica se relaciona con lo encontrado en la literatura hasta el momento ${ }^{16}$.

"Mire, salve esa suturita, donde le llegue a faltar otro punto, mire a ver cómo se las arregla, porque es la única que nos queda"' o: "'Mandemos al familiar a la farmacia para ver si consigue este equipo de venoclisis, de este suero porque no tenemos", en muchos sitios de subsidiado, se ve la ausencia de insumos, entonces pues obviamente eso deteriora también, es una desigualdad $y$ deteriora pues la misma calidad, y pues una atención segura y de calidad para la materna, entonces la parte de insumos, claro, porque tiene que uno a veces que hacer peripecias para hacer rendir una suturita que era la única que quedaba en el turno, donde se fuera un punto de más, pues ya quedamos maniatados" (obstetra régimen subsidiado y prepagada).

Los modos de vida de las mujeres (lugar de la vivienda, lugar del trabajo, lugar de los servicios de salud, condiciones de la vivienda, relaciones de género, capacidad de pagar por el trabajo que ellas realizan, etc.), explotación laboral, asociación en defensa de derechos laborales, relación con las jerarquías laborales y exigencias de producción laboral fueron temas presentes en los resultados, sin embargo tienen mayor relación con el Proceso Salud Enfermedad como se ha planteado en investigaciones previas ${ }^{17-19}$.

En la presente investigación hubo aspectos de clase social (exigencias de producción laboral) que mostró relación con la afectación de la salud mental, sobre todo de las mujeres asalariadas, la cual se relaciona con complicaciones biológicas en el proceso de EPP.

"se ha visto bastante presión por la parte de los directivos, hacia toda el área en general, ha sido mucho estrés a nivel laboral, han despedido personas. Cada día que pasa es más estresante, ya quiero estar en vacaciones. Ahorita ya estamos en la clínica porque la niña quiere nacer antes de tiempo" (mujer profesional asalariada régimen contributivo).

Lo anterior tiene relación con publicaciones previas, las cuales mostraron que el estrés en el trabajo está asociado a bajo peso al nacer ${ }^{20,21}$. Aunque este tema no 
hizo parte del objetivo de la presente investigación, es importante en salud pública y se propone como futura línea de investigación.

Por otro lado, la conceptualización de clase social es insuficiente para comprender la afectación de la salud de las mujeres dedicadas al trabajo doméstico y reproductivo, al trabajo informal, e independiente, para lo cual se proponen futuras líneas de investigación que incluyan estos aspectos bajo otras teorías diferentes a la de clase social (teoría colonialidad y de género).

El seguro autoriza (Figura 2).



Figura 2. El seguro autoriza.

El proceso de trabajo y la relación con los medios de producción de las mujeres, relacionado con la clase social, determina el régimen de afiliación en salud.

Las condiciones de acceso a los servicios de salud facilitan o no el servicio a especialistas, hacer la fila para esperar la atención, la disponibilidad de citas y agenda para programar la cita, perder la cita habiendo llegado al servicio, la prioridad que le dan las instituciones a las maternas, madrugar para conseguir atención, pedir la cita, las condiciones de la espera y elegir la clínica o el personal. Todos estos aspectos presentan desigualdades según la clase social viéndose afectadas las mujeres con una clase social menos aventajada.

"La fila preferencial para atención de gestantes se pregona en todas las actividades, pero las pacientes cuando llegan a la consulta con uno, le muestran que han tenido que madrugar a la consecución de su cita, no han encontrado la fila preferencial, la persona que la atiende la ha puesto a esperar a pesar de su condición de gestante, no hay sillas para que permanezcan sentadas mientras esperan, permanecen de pie, en los dias de lluvia a veces están haciendo fila en sitios donde ellas se mojan y, pues, una serie de cosas que si uno va a ver en el plan de acción que se hizo, el papel aguanta todo pero la práctica no lleva a eso" (obstetra régimen subsidiado)

"igual se hubiera demorado por ahí unos 15 dias o un mes en que me atendieran otra vez, ya muy tarde, entonces en vista que las citas no son tan rápida, no son tan cercanas, pues me toca google" (mujer trabajo informal, régimen subsidiado).

"para pedir cita me toca por teléfono, y llamo y en el call center las muchachas me atienden muy bien y todo pero nunca hay agenda en él, pues en él, la sede que me queda más cercana nunca hay agenda, no hay ese tipo de citas, no hay médico general, entonces me las dan pero para unas zonas muy lejanas y pues no puedo, me da miedo irme en el transporte público con el niño, entonces no, por ese lado no he podido, y la otra es seguir llamando, pero entonces me da malgenio porque me tienen hasta una hora y media ahi en el teléfono y pa que me digan que no" (Mujer trabajo informal, régimen subsidiado).

Trámites administrativos: son todas las actividades que hacen las maternas en las instituciones para acceder a los servicios, como las autorizaciones, la facturación, la solicitud de servicios, el proceso de afiliación, el cambio de un régimen a otro, el cambio de seguro o Empresa Promotora de Salud (EPS).

"Para afiliar la bebé a la prepagada es un trámite. La asesora me dijo que tenía que hacerlo entre la semana 18 y la 22 creo, ehhh hice los tramites, eran una y un certificado médico, cómo yo no estaba aquí, pues envié la ecografía que me habia hecho allá y el certificado médico me hizo el favor de solicitárselo a mi ginecólogo. Mi hermano pidió una cita normal, la pagamos, él le comentó, le dieron el certificado, ya, ehhh el trámite se demoró un montón y después la asesora no me decía nada nunca, y yo le escribía y esta señora no se daba por enterada y después de un tiempo me dijo que habian rechazado la solicitud porque la ecografia no era de una de las instituciones que estaba afiliada al directorio del plan complementario, entonces me tocó hacer una carta explicando que yo me encontraba fuera del país todo eso, bueno se 
demoraron otro montón de tiempo, lo mismo, a bueno y desde muy temprano le pregunte cual era el motivo por el que yo tenía que afiliar a mi bebé desde la semana veinte y pico y empezar a pagar por un bebé que no había nacido, yo no entendía eso, no me respondió nunca" (mujer profesional con especialización, independiente, plan complementario).

Afectación de la salud por las barreras de acceso: incluye la no prestación de servicios de salud poniendo en riesgo la salud de la mujer o el hijo y sometiendo a las usuarias a trámites para solicitar los derechos. Por ejemplo, la imposibilidad de asistir al control prenatal porque la EPS no autoriza la atención en otra ciudad, la ausencia de servicios relacionados con los derechos de la ruta materno perinatal (curso de preparación para la maternidad y la paternidad), la no autorización de la licencia maternidad por parte de la EPS.

"yo pedi fue una portabilidad, o sea, entonces a mí me dijeron que cuántos días, que cuánto iba a durar en Bogotá, entonces ella me dijo que la portabilidad era por ahi para 7 meses, que si me quedaba acá en Bogotá viviendo, que entonces y a me tendría que afiliar a otra EPS o a otra cosa que hubiera acá en Bogotá. Pues yo habia hecho ya la vuelta en el EPS de Bogotá, me habían dicho que: Ay, que todavía no podían, que tenian que seguir por urgencias solamente, con la EPS de mi pueblo porque ellos todavía no podían, que eso demoraba por ahi unos tres meses para que me afiliaran que entonces ellos no podian hacer nada ni nada de eso, entonces me tocó pedir la portabilidad pero ya para qué los controles prenatales? si el bebé ya habia muerto después de 5 meses de estar embrazada sin atención en Bogotá" ( mujer trabajo informal régimen subsidiado).

En las anteriores categorías no se evidenciaron diferencias en los discursos de las mujeres y del personal de salud. En todas las mujeres participantes se presenció la violación de derechos sexuales y reproductivos. Ninguna de las mujeres participantes en la investigación recurrió a medidas legales para reclamar los derechos. Una de ellas (mujer profesional con estudios superiores) colocó la queja en la institución. Sin embargo, se pudo estar en contacto con algunas mujeres no participantes que han colocado derechos de petición en las instituciones de salud. No se lograron obtener las cifras a nivel nacional de las mujeres en EPP que recurren a mecanismos legales para hacer valer sus derechos, lo cual es relevante para la categoría de "itinerarios burocráticos"14 elegida en el marco referencial.

\section{Discusión}

En etnografía crítica es relevante el contexto donde adquieren sentido los discursos ${ }^{22}$. Al respecto, en Colombia sectores académicos y de la sociedad civil han participado para defender el derecho a la salud como un derecho fundamental y no como un servicio que se adquiere según la capacidad de pago de las personas. Por eso se han realizado propuestas para transformar el sistema de salud colombiano ${ }^{23}$ y al respecto se aprobó una ley en el Congreso de la Republica (Ley Estatutaria de la Salud del 2015). A pesar de lo anterior, se ha evidenciado incumplimiento a lo plasmado en la ley y por lo tanto se requieren reformas más estructurales que modifiquen el funcionamiento general del sistema, postulado en la Ley 100, y sus mecanismos de financiación ${ }^{24,25}$.

Según un informe de la Secretaria de Salud de Bogotá, en el régimen subsidiado (2015) las barreras de acceso más frecuentes fueron: 41,5\% dificultad de accesibilidad administrativa, $18,9 \%$ inconsistencias en el sistema de información-aseguramiento y $17 \%$ no oportunidad de servicios. También se presentó atención deshumanizada, dificultades relacionadas por el alto costo del servicio, dificultad por tutelas, negación de servicios, y no suministro de medicamentos. De las tres localidades con mayores dificultades dos pertenecían al sur de Bogotá donde se encuentra la población más vulnerable ${ }^{3}$.

Por otro lado, existen otras fallas del sistema de salud colombiano relacionadas con el concepto de salud como mercancía ${ }^{23}$ que promociona como beneficio la libre elección del usuario de las instituciones de salud, lo cual en teoría mejoraría la calidad de la atención. Lo anterior se opone a la salud como bien público donde "los servicios no son rivales en consumo ni excluyentes" ${ }^{\prime 25}$.

En el contexto de la salud como negocio se crean monopolios de prestación de servicios que no benefician a toda la población por igual, y la información que tiene el usuario es diferente a la del prestador y éste puede beneficiarse para generar más rentabilidad. Aunque el Estado debe controlar esta falla del mercado, la corrupción ha desviado recursos públicos de la salud con fines comerciales, y las funciones de vigilancia y control quedan minimizadas ${ }^{23-25}$.

Otros problemas son que los usuarios desconocen los derechos, tienen temor a cambiar de seguro de salud, y los empleadores eligen la EPS para el caso de los asalariados. El seguro regula dónde se remiten los 
pacientes, eligen las clínicas y hospitales para disminuir costos. Los alcaldes de los municipios contratan una sola aseguradora ${ }^{25}$.

Los discursos y el contexto permiten que emerjan otras teorías en relación con los resultados. Al respecto, las desigualdades según clase social se pueden analizar desde la teoría de la violencia estructural. Singer retoma a Galtung y define violencia estructural como "una etiqueta social impuesta que constriñe el potencial humano generado por la prevalencia de estructuras económicas y políticas como acceso desigual a los recursos que se necesitan para sostener la vida o proveer una razonable calidad de vida, restringe la adquisición de poder político, niega iguales oportunidades para la educación y discrimina en otras esferas de la vida diaria". La inequidad en salud es una forma de violencia estructural ${ }^{19}$.

Bellamy y Castro retoman a Galtung, Farmer, Scheper Hughes y Bourgois exponen la violencia institucional que comparte características de la violencia estructural. En este sentido no hay acciones deliberadas de mala fe, se intersecciona con la exclusión social y las prácticas violentas son invisibles con una intención definida, donde "las personas aprenden a ser pacientes del Estado"26.

Las categorías que comprenden este tipo de violencia, en los servicios de urgencias públicos de México son: la espera (incluye la incertidumbre y la arbitrariedad), la producción institucional de la sujeción (vigilancia y control para mantener el orden de los individuos por fuera y por dentro de la institución) y la alienación al derecho de la salud (lógica del favor, súplica de atención y agradecimiento si son atendidos, no se reclaman derechos, volver al mismo servicio porque no hay otra opción) ${ }^{26}$. La anterior conceptualización es coherente con los resultados de la presente investigación.

Referente a la conceptualización de acceso se han desarrollado varias teorías. Entre ellas el "modelo de cobertura efectiva" de Tanahashi. Esta teoría dialoga con los sistemas de salud que son regulados por el mercado, e ignora la visión ética del derecho a la salud. Por lo cual Arrivillaga y Borrero proponen otros modelos como los de Ruger, Braveman y Gruskin que dan cuenta de los modos de la vida de las personas y su relación con el Proceso de Salud Enfermedad y Atención (PSEA) de la población ${ }^{27}$. Hacer etnografía es una herramienta metodológica relevante en salud pública que permite dar cuenta de lo anterior y desarrollar en compañía de las personas que participan en el trabajo de campo los marcos referenciales de los derechos humanos.
Algunas de las limitaciones operativas y metodológicas de la presente investigación fueron discutidas previamente ${ }^{10}$, sin embargo, es relevante también presentar algunas de las limitaciones teóricas.

La categorización de clase social se realizó teniendo en cuenta la teoría de clase social de Breilh ${ }^{12,13}$. Desde esa perspectiva el proceso de trabajo de las mujeres relacionado con la clase social determina el régimen de afiliación en salud. Sin embargo, según otros autores como Wright y Bourdieu, se tienen en cuenta otros elementos para conceptualizar la clase social. En ese contexto existen mujeres de diferente clase social con trabajo asalariado, que pertenecen al régimen contributivo. Teniendo en cuenta lo anterior no se logró la saturación teórica pues hubiera sido posible que participaran más mujeres en la investigación. Sin embargo, por cuestiones logísticas de tiempo y presupuesto no fue posible.

Wright conceptualiza la clase social a través de 3 mecanismos centrales de clase: la propiedad, el control y el credencialismo ${ }^{18}$. La propiedad y el control son conceptos relacionados con las categorías expuestas por Breilh ${ }^{13}$ y desarrolladas en la investigación. Sin embargo, el concepto de Credencialismo no es desarrollado por Breilh. A pesar de lo anterior, es posible visibilizar en los itinerarios de acompañamiento cómo determinadas cualificaciones y credenciales educativas determinan el acceso exclusivo a mercados de trabajo y empleo específicos, lo cual posibilita tener mejores ingresos económicos. Por ejemplo, las credenciales de la mujer empresaria no solo le permite tener mejores ingresos económicos y acceso a servicios privados en salud, sino poder tomar decisiones sobre su PSEA.

La construcción de clase social, según Bourdieu, requiere definir no solo los diferentes capitales sino los conceptos de campo y habitus. Para lograr todo lo anterior es necesario focalizar el trabajo de campo en un espacio definido y delimitado ${ }^{28}$ lo cual es diferente a lo encontrado en el trabajo de campo de la presente investigación que requirió espacios de trabajo heterogéneos.

El capital social se refiere a las relaciones sociales que tienen los diferentes agentes sociales, el capital cultural se refiere a lo aprendido en la casa y en el colegio, el capital simbólico que es el reconocimiento social que se le da al capital y el capital económico que se expresa a través de dinero, y requiere de tiempo libre para convertirse en otras formas de capital ${ }^{29}$. 
La salud pública ha intentado abstraer de manera aislada los conceptos de Bourdieu como es el caso del capital social en relación con la salud ${ }^{30}$, lo cual también es posible evidenciar en los itinerarios de acompañamiento de la presente investigación que muestran las ventajas que tienen las mujeres cuando pertenecen a determinadas redes sociales, no solo familiares sino también las relacionadas con el parto humanizado. Igualmente, en los itinerarios es posible visibilizar el contexto familiar, educativo y económico de las mujeres y como éstos son o no son reconocidos en los servicios de salud o por sus redes sociales. Lo anterior se relaciona con el poder que tienen las participantes en las instituciones de salud. En ese sentido las mujeres que pertenecen a redes sociales como las de parto humanizado, amigos que pertenecen al sistema de salud o son abogados logran mayor autonomía en los servicios de salud.

Se concluye que acompañar las mujeres en EPP desde un paradigma hermenéutico crítico, es tener la oportunidad de acompañar un proceso histórico, así mismo, es un desafío que requiere inicialmente enunciar los discursos, deconstruirlos, y transformar racionalidades. Todo lo anterior son aspectos necesarios para que las prácticas sociales se transformen. Por lo cual es necesario integrar las investigaciones empírico analíticas con investigaciones que den cuenta de los significados del lenguaje y posibles trasformaciones desde esta perspectiva.

Se recomienda fomentar investigaciones en salud pública integrales que incluyan los temas de derechos y deberes, incluyendo los elementos de la Ley Estatuaria y los conceptos expuestos en la investigación relacionados con la violencia estructural e institucional. Es importante generar un acompañamiento a las mujeres en EPP y personal de salud que deseen conocer sus derechos y hacerlos valer en los servicios de salud. Este acompañamiento requiere apoyo legal para transformar las instituciones en el sentido de la humanización.

Se necesitan futuras investigaciones que fortalezcan los comités de participación comunitaria en salud (COPACOS), las ligas de usuarios, entre otras, y hacer cumplir la ley en lo referente a la información al usuario, y a la libre selección de la EPS por parte de la trabajadora (or).

\section{Agradecimientos}

A Colciencias convocatoria 647 y la convocatoria pequeña cuantía de la Universidad CES de Medellín por la financiación. Mujeres y personal de salud que participaron en la investigación, Fundación Arka, 292
Organización Apapachoa, Movimiento Nacional por la Salud Sexual y Reproductiva en Colombia, Tribu Criarte, Secretaria de Salud Bogotá. A Marcelo Amable, Alfredo Maya y Mónica Saenz por sus aportes desde las ciencias sociales.

\section{Consideraciones éticas}

Se contó con aprobación del comité de ética de la Universidad CES (Acta No 99/2016) y de la Secretaria de salud de Bogotá (Acta N0041000/2017).

\section{Conflictos de interés}

Los autores declaran no tener conflicto de interés.

\section{Referencias}

1. Ministerio de Salud de Colombia. Plan decenal de salud pública 2012-2021. Bogotá: Ministerio de Salud de Colombia 2012.

2. Ministerio de Salud y de la Protección Social de Colombia. Dirección de epidemiología y demografía. Análisis De Situación De Salud (ASIS) Colombia, 2017 Bogotá Ministerio de Salud y de la Protección Social de Colombia. Dirección de Epidemiología y Demografía; 2018.

3. Secretaría Distrital de Salud de Bogotá, Dirección de Planeación Sectorial, Subdirección de Vigilancia en Salud Pública, Grupo Análisis de Condiciones Calidad de Vida Salud y Enfermedad. Documento de Análisis de Situación de Salud con el Modelo de los Determinantes Sociales de Salud para el Distrito Capital Bogotá Secretaría Distrital de Salud de Bogotá; 2017.

4. Secretaría distrital de salud de Bogotá. Direccion de salud pública. Mortalidad Materna Bogotá. In: Secretaria distrital de salud de Bogotá. Dirección de salud pública, editor. Unidad de Análisis sobre Salud Materna y Perinatal; Fundación Santa Fé de Bogotá 2016.

5. Dirección de salud pública. Mortalidad materna Bogotá Secretaría distrital de salud de Bogotá; 2012. http://www.saludcapital.gov.co/sitios/ VigilanciaSaludPublica/Protocolos $\% 20$ de $\% 20$ Vigilancia\%20en\%20 Salud\%20 Publica/ Mortalidad\%20Materna.pdf.

6. Oróstegui R, Bermudez C, Linares A, Rueda M, Gonzales J, Arevalo T. Informe de calidad de vida de Bogotá Bogotá: Programa "Bogota como vamos" 2017. http://www.bogotacomovamos. org/documentos/informe-de-calidad-de-vida-enbogota-en-2017/. 
7. Aguado Quintero LF, Girón Cruz LE, Osorio Mejía AM, Tovar Cuevas LM, Ahumada Castro JR. Determinantes del uso de los servicios de salud materna en el Litoral Pacífico Colombiano. Rev Latinoam Cienc Soc Niñez Juv. 2007; 5(1): 233-281.

8. Ramírez HS. Calidad de la atención en salud. Prácticas y representaciones sociales en las poblaciones quechua y aymara del antiplano boliviano. La Paz: OPS; 2010.

9. Bedoya-Ruiz LA, Agudelo-Suárez AA. Relación de las mujeres en embarazo parto y postparto (EPP) con los servicios de salud según la clase social. Rev Gerenc Polít Salud. 2019; 18(36). doi: http://dx.doi. org/10.17843/rpmesp.2020.371.4963

10. Bedoya-Ruiz LA, Agudelo-Suárez AA, RestrepoOchoa DA. Relaciones que establecen las mujeres durante el embarazo, parto y posparto con el personal de salud según clase social en Bogotá: estudio cualitativo. Rev Peru Med Exp Salud Publica. 2020; 37(1): 7-16. doi: https://doi.org/0.17843/ rpmesp.2020.371.4963

11. Kincheloe J, McLaren P. Replanteo de la teoría crítica y de la investigación cualitativa In: Denzin NK, Lincoln YS, editors. Manual de investigación cualitativa Volumen II Paradigmas y perspectivas en disputa Barcelona Gedisa; 2012. p. 241-291.

12. Breilh J. Replanteo del método epidemiológico In: Breilh J, editor. Crítica a la interpretación capitalista de la Epidemiología Un ensayo de desmitificación del proceso de salud y enfermedad. Mexico: Universidad Autonoma Metropolitana de Xochimilco; 1977.

13. Breilh J. Breverecopilación sobre operacionalización de la clase social para encuestas en la investigación social. In: Centro de estudios y asesoría en salud (CEAS), editor. Quito. Ecuador Centro de estudios y asesoría en salud (CEAS); 1989. p. 1-12.

14. Abadía Barrero CE, Oviedo Manrique DG. Itinerarios burocráticos de la salud en Colombia: la burocracia neoliberal, su estado y la ciudadanía en salud. Rev Ger Polít Salud. 2010; 9: 86-102. doi: https://doi.org/10.11144/Javeriana.rgsp9-18.ibsc

15. Restrepo-Ochoa DA. Vigencia de la categoría clase social en salud pública In: Estrada M J H, editor. Teoría crítica de la sociedad y salud pública. Bogotá: Universidad Nacional de Colombia. Universidad de Antioquia; 2011. p. 134-144.

16. Ministerio de Salud de Colombia. Salud materno infantil In: Ministerio de Salud de Colombia, editor. Encuesta Nacional de Demografía y salud Bogotá Profamilia 2015.

17. Instituto Nacional de salud, Ministerio de salud de Colombia. Clase social y salud Bogotá: Instituto Nacional de salud, Observatorio Nacional de Salud,
Ministerio de salud de Colombia 2016. https:// www.ins.gov.co/Direcciones/ONS/Informes/8.\%20 Clase\%20Social\%20y\%20Salud.pdf.

18. Rocha KB, Muntaner C, Solar O, Borrell C, Bernales P, González MJ, et al. Clase social, factores de riesgo psicosocial en el trabajo y su asociación con la salud autopercibida y mental en Chile. Cad Saúde Pública. 2014; 30: 2219-2234. doi: https:// doi.org/10.1590/0102-311X00176213.

19. Singer M. Capítulo 6. Inequity as a cofactor The syndemic impact of social disparities. In: Singer M, editor. Introduction to Syndemics. San Francisco: Jossey Bas 2009. p. 121-156.

20. Marrero Santos MdL, Román Hernández JJ, Salomón Avich N. Estrés psicosocial laboral como factor de riesgo para las complicaciones de la gestación y el bajo peso al nacer. Rev Cubana de Salud Pública. 2013; 39(1): 864-880.

21. Taboada Aguirre E, Manzanera Balderas E, Dávalos Torres MM. Condición laboral asociada a complicaciones en el embarazo en noreste de México. Med Segur TraB. 2014; 60(237): 612-619. doi: http://dx.doi.org/10.4321/S0465546X2014000400002

22. Scheper-Hughes N. Nervoso In: Scheper-Hughes $\mathrm{N}$, editor. La muerte sin llanto Violencia y vida cotidiana en Brasil. Barcelona: Ariel 1997.

23. Alianza nacional por un nuevo sistema de salud, Comisión de Seguimiento a la Sentencia T - 760 de 2008 y de Reforma Estructural al Sistema de Salud y Seguridad Social, Viva la ciudadanía. Salud: Iniciativas legislativas desde la Sociedad civil. Bogotá: CSR,ANSA; 2014.

24. Jiménez WG, Angulo LL, Castiblanco YP, Gómez ML, Rey LJ, Solano LT, et al. Ley estatutaria: ¿avance hacia la garantía del derecho fundamental a la salud? Rev Col Cirugía. 2016; 31(2): 81-90.

25. Bejarano-Daza JE, Hernández-Losada DF. Fallas del mercado de salud colombiano. Rev Fac Med. 2017; 65(1): 107-113. doi: http://dx.doi.org/10.15446/ revfacmed.v65n1.57454

26. Bellamy C, Castro R. Formas de violencia institucional en la sala de espera de urgencias en un hospital público de México. Rev Cienc Salud. 2019; 17(1):18. doi: https://doi.org/10.12804/revistas. urosario.edu.co/revsalud/a.7621

27. Arrivillaga M, Borrero YE. Visión comprensiva y crítica de los modelos conceptuales sobre acceso a servicios de salud, 1970-2013. Cad Saúde Pública. 2016; 32(5). http://dx.doi.org/10.1590/0102$311 \times 00111415$

28. Castro R, José Suárez HJ. Introduccion: trabajar con Pierre Bourdieu. In: Castro R, José Suárez 
HJ, editors. Pierre Bourdieu en la sociología latinoamericana: el uso de campo y habitus en la investigación Cuernavaca, Morelos: Universidad Nacional Autónoma de México, Centro Regional de Investigaciones Multidisciplinarias; 2018. p. 11-25.

29. Martínez GJS. Las clases sociales y el capital en Pierre Bourdieu un intento de aclaración. Salamanca Universidad de Salamanca Departamento de Sociología; 1994.
30. Martínez-Herrera E, Moreno-Mattar O, Dover RVH. El significado del capital social "individual" en diabéticos receptores de cuidado en un contexto urbano colombiano. Cad Saúde Pública. 2015; 31(4): 837-849. doi: http://dx.doi.org/10.1590/0102$311 \times 00167113$

Tabla 1. Características sociodemográficas mujeres gestantes participantes en la investigación. Bogotá.

\begin{tabular}{|c|c|c|c|c|c|c|c|c|}
\hline Mujer & $\begin{array}{l}\text { Régimen } \\
\text { salud }\end{array}$ & $\begin{array}{r}\text { Edad } \\
\text { (años) }\end{array}$ & $\begin{array}{c}\text { Semanas } \\
\text { gestación } \\
\text { inicio } \\
\text { estudio } \\
\end{array}$ & Ocupación & Nivel educativo & $\begin{array}{l}\text { Estado } \\
\text { civil }\end{array}$ & $\mathbf{E S E}^{\mathbf{a}}$ & $\begin{array}{l}\text { Fuente } \\
\text { ingresos }\end{array}$ \\
\hline Gp & Pre pagada & 38 & 27 & $\begin{array}{c}\text { Empresaria } \\
\text { servicios privados } \\
\text { medicina estética }\end{array}$ & $\begin{array}{l}\text { Profesional Medicina } \\
\text { Post grado }\end{array}$ & casada & 5 & $\begin{array}{l}\text { Trabajo (de } \\
\text { ella, pareja) y } \\
\text { propiedades }\end{array}$ \\
\hline Ap & $\begin{array}{c}\text { Plan } \\
\text { complementario } \\
\mathrm{C}^{\mathrm{b}}\end{array}$ & 32 & 8 & Ama de casa & Profesional Ingeniería & casada & 3 & $\begin{array}{l}\text { Trabajo pareja } \\
\text { y propiedades }^{\mathrm{c}}\end{array}$ \\
\hline Lp & $\begin{array}{c}\text { Plan } \\
\text { complementario } \\
\mathrm{S}\end{array}$ & 34 & 32 & $\begin{array}{l}\text { Trabajo informal } \\
\text { investigadora }\end{array}$ & $\begin{array}{c}\text { Profesional } \\
\text { Antropología Post } \\
\text { grado }\end{array}$ & $\begin{array}{l}\text { unión } \\
\text { marital de } \\
\text { hecho }\end{array}$ & 3 & $\begin{array}{l}\text { Trabajo de ella, } \\
\text { pareja, padre }\end{array}$ \\
\hline $\mathrm{Kc}$ & Contributivo $\mathrm{S}$ & 27 & 28 & Asalariada & Profesional Ingeniería & casada & 3 & $\begin{array}{c}\text { Trabajo de ella, } \\
\text { pareja }\end{array}$ \\
\hline Ac & Contributivo N & 23 & 16 & Asalariada & $\begin{array}{l}\text { Técnica en gestión de } \\
\text { archivo }\end{array}$ & casada & 2 & $\begin{array}{c}\text { Trabajo de ella, } \\
\text { pareja }\end{array}$ \\
\hline $\mathrm{Lc}$ & Contributivo $\mathrm{C}$ & 18 & 20 & Ama de casa & Bachiller & soltera & 2 & $\begin{array}{c}\text { Trabajo, padres, } \\
\text { hermanos }\end{array}$ \\
\hline Ss & Subsidiado ES ${ }^{d}$ & 19 & 25 & Ama de casa & Séptimo bachillerato & $\begin{array}{l}\text { Unión } \\
\text { marital de } \\
\text { hecho }\end{array}$ & 1 & $\begin{array}{l}\text { Trabajo pareja, } \\
\text { suegra }\end{array}$ \\
\hline Gs & Subsidiado ES & 36 & 20 & $\begin{array}{c}\text { Trabajo informal } \\
\text { varios }\end{array}$ & Octavo bachillerato & soltera & 1 & Trabajo de ella \\
\hline Vs & Subsidiado $\mathrm{FF}^{\mathrm{e}}$ & 27 & 38 & Ama de casa & Bachiller & soltera & 1 & Trabajo padres \\
\hline
\end{tabular}

a. Estratos socio económicos. Estos en Colombia son 6, siendo 1 el más bajo y 6 el más alto. Esta estratificación se realiza de acuerdo al lugar de la vivienda. b A pesar que dos mujeres tienen planes complementarios las empresas que prestan los servicios son diferentes y por lo tanto las clínicas de atención son diferentes. Lo anterior también sucede en el régimen contributivo y subsidiado. Por lo tanto, se coloca la inicial para visibilizar las empresas que son diferentes. c. Familias que tienen capacidad de ahorro y reciben de ingresos de otras fuentes diferentes al trabajo (arriendos, alquiler de equipos). d. EPS subsidiada. E. Fondo Financiero.

Tabla 2. Características del personal de salud con experiencia en atención materna participante del estudio. Bogotá.

\begin{tabular}{|c|c|c|c|c|}
\hline Tipo & Lugar de trabajo & Sexo & Edad & $\begin{array}{l}\text { Experiencia laboral en } \\
\text { maternidad (años) }\end{array}$ \\
\hline $\begin{array}{l}\text { Obstetra atención de partos, consulta externa, } \\
\text { preparación parto }\end{array}$ & prepagada & $\mathrm{F}$ & 48 & 18 \\
\hline Asesora de lactancia materna & prepagada y contributivo & $\mathrm{F}$ & 41 & 21 \\
\hline Enfermera consulta externa & prepagada y contributivo & $\mathrm{F}$ & 28 & 4 \\
\hline Medica atención de partos, consulta externa & contributivo y subsidiado & $\mathrm{F}$ & 40 & 5 \\
\hline Obstetra consulta externa & subsidiado & M & 54 & 25 \\
\hline Obstetra atención de partos, consulta externa & subsidiado & $\mathrm{F}$ & 32 & 8 \\
\hline $\begin{array}{l}\text { Enfermera consulta externa y preparación } \\
\text { embarazo parto y post parto }\end{array}$ & subsidiado & $\mathrm{F}$ & 46 & 15 \\
\hline $\begin{array}{l}\text { Fisioterapeuta atención de partos, preparación } \\
\text { embarazo, parto y post parto }\end{array}$ & contributivo y prepagada & $\mathrm{F}$ & 33 & 5 \\
\hline
\end{tabular}

